\title{
Langfristiger Nutzen der Chemotherapie
}

Eine Chemotherapie wirkt bei Patienten mit anaplastischem Gliom nach der Op. zwar ähnlich gut wie die Bestrahlung, mit der Radiatio ist das Gesamtüberleben langfristig aber tendenziell besser. $\mathrm{Zu}$ dem kann Temozolomid nicht in allen prognostischen Subgruppen die Chemotherapie ersetzen. Das zeigte die Langzeitauswertung der NOA-04-Studie.

In der 2009 veröffentlichten Studie wurde bei 318 Patienten mit einem anaplastischen Gliom im Grad III eine Radiotherapie gegen eine Chemotherapie nach der Op. verglichen. Letztere erfolgte entweder mit Temozolomid (TMZ) oder nach dem PCV-Schema (Procarbazin, CCNU, Vincristin). Dabei war eine Chemotherapie nach der Op. beim anaplastischen Gliom der Radiatio ebenbürtig.
Inzwischen liegt eine Auswertung nach im Median 9,5 Nachbeobachtungsjahren vor. Im Wesentlichen wurden die Ergebnisse der primären Analyse bestätigt: $\mathrm{Zu}$ einer Progression kam es mit der Radiatio als erster Option bei $78 \%$ der Patienten, mit der Chemotherapie bei 79\%. Ein Therapieversagen stellten die Ärzte bei jeweils 66 und $67 \%$ der Patienten nach im Median 4,4 und 4,6 Jahren fest.

Auch beim Gesamtüberleben fanden die Studienautoren in beiden Therapiearmen keine statistisch signifikanten Unterschiede (52 vs. $47 \%$ ), wenngleich die Überlebensrate mit Radiatio als erster Therapiewahl nach der Op. relativ betrachtet um $11 \%$ höher lag.

In den Chemotherapiegruppen gab es insgesamt keine statistisch belastbaren

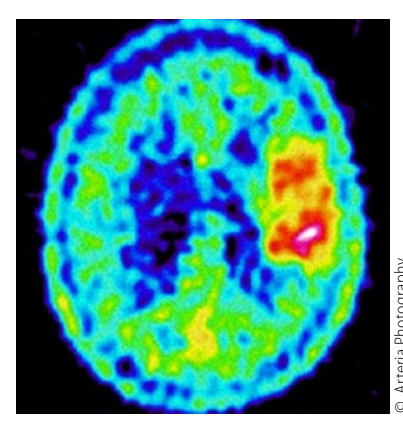

Bei einem Gliom sind Chemotherapie und Bestrahlung vergleichbar effektiv.

Unterschiede beim progressionsfreien Überleben zwischen einer Behandlung mit PCV (2,5 Jahre) und TMZ (2,7 Jahre). Patienten mit Gliom-CpG-Inseln-Methylierungs-Phänotyp (G-CIMP) und 1p/ 19q-Kodeletion lebten jedoch mehr als doppelt so lange progressionsfrei, wenn sie PCV statt TMZ erhielten (9,4 vs. 4,5 Jahre). Auch die Radiatio war hier TMZ deutlich überlegen.

Thomas Müller

Wick W et al. Long-term analysis of the NOA-04 randomized phase III trial of sequential radiochemotherapy of anaplastic glioma with PCV or temozolomide. Neuro Oncol. 2016;18(11);1529-37.

\section{Analkarzinom}

\section{Ansprechen kann sich stark verzögern}

Bei Patienten mit einem Analkarzinom reicht es möglicherweise aus, erst 26 Wochen nach Beginn einer Chemoradiotherapie das Ansprechen auf die Behandlung zu überprüfen - und nicht bereits nach elf Wochen. Gemäß den Ergebnissen der britischen ACT-II-Studie zufolge dauert das Therapieansprechen bei einigen Pati- enten offenbar deutlich länger als die in Leitlinien empfohlene Zeitspanne.

An der Studie hatten knapp 1.000 Patienten mit neu diagnostiziertem, histologisch bestätigtem Analkarzinom teilgenommen. Sie wurden intravenös mit Mitomycin oder Cisplatin behandelt. Zudem erhielten sie ebenfalls intravenös

\section{kurz notiert}

\section{Tumorbekämpfung mit Adenoviren}

Spanische Wissenschaftler haben onkolytische Adenoviren entwickelt, die hochspezifisch gegen Tumorzellen wirken, ohne gesundes Gewebe zu schädigen. Dabei machten sie sich die unterschiedliche Expression von (PEB-Proteinen (CPEB: "cytoplasmic polyadenylation element binding") in normalen und malignen Zellen zunutze. CPEB ist eine Familie von vier RNA-bindenden Proteinen, die die Genexpression kontrollieren. CPEB4 wird in Tumorzellen stark exprimiert, es ist für das Tumorwachstum notwendig. CPEB1 wird in normalem Gewebe exprimiert, aber nicht in Tumorzellen. Die Wissenschaftler haben in das Genom von Adenoviren eine Sequenz eingebaut, die CPEB-Proteine erkennt. Diese modifizierten Adenoviren greifen nur solche Zellen an, die hohe CPEB4- und niedrige CPEB1-Spiegel aufweisen - also nur Tumorzellen. Diese onkoselektiven Viren werden durch CPEB4 aktiviert und durch CPEB1 unterdrückt. Dies führt dazu, dass die Wirkung in Tumorzellen vorhanden, in gesunden Zellen aber abgeschwächt ist. Sobald die Viren in Tumorzellen eindringen, wird ihr Genom repliziert. Wenn sie die Tumorzelle verlassen, zerstören sie die Zelle und lassen weitere Viruspartikel zurück, die weitere Tumorzellen infizieren können. Ihre Wirksamkeit wurde in In-vitro-Modellen von Pankreaskrebs und in Mausmodellen untersucht [Villanueva E et al. Nat Commun. 2017;8:14833].

Judith Neumaie
Fluorouracil sowie eine Bestrahlung (50,4 Gy über 28 Tage). Die mediane Gesamtbehandlungsdauer betrug 38 Tage.

Ein komplettes klinisches Ansprechen wurde nach elf Wochen bei $52 \%$ der 940 Studienteilnehmer dokumentiert, nach 18 Wochen bei $71 \%$ und nach 26 Wochen bei $78 \%$. Insgesamt 691 Patienten nahmen an allen drei Untersuchungen teil. Nach elf Wochen wurde in dieser Subgruppe bei $64 \%$ ein komplettes klinisches Ansprechen festgestellt, nach 18 Wochen bei $80 \%$ und nach 26 Wochen bei $85 \%$.

Die 5-Jahres-Gesamtüberlebensraten für Patienten mit kompletter Response zu den genannten drei Zeitpunkten, die an allen drei Untersuchungen teilgenommen hatten, betrugen $85 \%, 86 \%$ und $87 \%$. Die Raten für das 5-Jahres-Gesamtüberleben von Patienten ohne komplettes Ansprechen lagen bei $72 \%, 59 \%$ und $46 \%$.

Auch das progressionsfreie Überleben unterschied sich in der Gesamtstudiengruppe zwischen denen mit und denen ohne komplettes Ansprechen (75 vs. $63 \%$, 75 vs. $53 \%$ bzw. 80 vs. $33 \%$ ). Peter Leiner

Glynne-Jones R et al. Best time to assess complete clinical response after chemoradiotherapy in squamous cell carcinoma of the anus (ACT II): a post-hoc analysis of randomised controlled phase 3 trial. Lancet Oncol. 2017;18(3):347-56. 